\title{
Co-prime Probability for Nonabelian Metabelian Groups of Order Less than 24 and Their Related Graphs
}

\author{
Nurfarah Zulkifli* and Nor Muhainiah Mohd Ali \\ Department of Mathematical Sciences, Universiti Teknologi Malaysia \\ 81310 UTM Johor Bahru, Malaysia \\ *Corresponding author: nurfarahzulkifli@yahoo.com
}

Article history

Received: 22 November 2018

Received in revised form: 17 May 2019

Accepted: 17 July 2019

Published online: 1 December 2019

\begin{abstract}
Let $G$ be a finite group. The probability of a random pair of elements in $G$ are said to be co-prime when the greatest common divisor of order $x$ and $y$ where $x$ and $y$ in $G$, is equal to one. Meanwhile the co-prime graph of a group is defined as a graph whose vertices are elements of $G$ and two distinct vertices are adjacent if and only if the greatest common divisor of order $x$ and $y$ is equal to one. In this paper, the co-prime probability and its graphs such as the types and the properties of the graph are determined.
\end{abstract}

Keywords Co-prime probability; co-prime graph; number of edges; types of graph; dominating number; independent number.

Mathematics Subject Classification $20 \mathrm{~K} 99$.

\section{Introduction}

A simple definition on probability is how likely something is to happen. Mathematically, the probability of an event to happen is a number of ways it happens divided by total number of the outcomes.

For the past few years, researches on commutativity degree focuses on nonabelian metabelian groups have been done. It started by Erdos and Turan [1] in 1968, whereby the research of commutativity degree focuses only on symmetric group, $S_{n}$. Later, this research has been further explored by studying the $n^{\text {th }}$ commutativity degree, the multiplicative degree, the submultiplicative degree, the relative commutativity degree and many more. In 2017, Abd Rhani [2] is then extended the commutativity degree of $G$ to co-prime probability of $G$. This probability is defined as the probability of the order of a random pair of elements in the group are relatively prime or co-prime. In Abd Rhani [2], the co-prime probability for all $p$ - groups and for some dihedral groups has been determined.

As for the graph part, the study of the co-prime graph for nonabelian metabelian groups of order less than 24 started with prime graph of $G$ introduced by Williams [3] in 1981. Later, the study of prime graph is then continuing to determine the co-prime graph of integers by 
Erdos and Sarkozy [4] and Sarkozy [5]. In 2014, Ma et al. [6] then extended the research by introducing the co-prime graph of $G$ for finite group. The types and some properties of the graphs were then determined. Unfortunately, none did a research on both co-prime probability and its graphs for nonabelian metabelian groups of order less than 24 .

In this paper, the co-prime probability for nonabelian metabelian groups of order less than 24 and their related graphs are discussed. The application of this research can also be seen in so many ways. It will give some contributions to the field of group and graph theory as this is a continuation study from the previous researchers. Graph theory also can be very useful in real life whereby the graph can be used as a solution to a problem. For example, in 1735, Konisberg bridge problem is solved by using the concept of planar and Eulerian graph. Another example of the application of graph is in business management whereby the star graph is related to a model named hub and spoke model. Therefore, in this paper, the co-prime probability for nonabelian metabelian groups of order less than 24 are discussed. Then, the graph for each group is identified. For each graph, the total number of edges, the types of graph and the properties of the graph such as the dominating number and the independent number are determined.

Hence, this paper is structured as follows: the first part is the introduction. The second part states the basic concepts, definitions, propositions and theorems that are used for this paper and lastly, the main results and the conclusion of this paper on both the co-prime probability and its graphs for nonabelian metabelian groups of order less than 24 are discussed.

\section{Preliminaries on Groups and Graphs}

In this section, some basic concepts on group and graph theory are stated.

Definition 1 [7] A group $G$ is metabelian if there exists a normal subgroup $A$ such that both $A$ and $G / A$ are abelian.

Definition 2 [8] Dihedral groups of degree $n$

For each $n \in \mathbb{Z}$ and $n \geq 3, D_{n}$ denoted as the set of symmetries of a regular $n-g o n$. Furthermore, the order of $D_{n}$ is $2 n$ or equivalently $\left|D_{n}\right|=2 n$. The Dihedral groups, $D_{n}$ can be represent in a form of generators and relations given in the following representation:

$$
D_{n}=\left\langle a, b \mid a^{n}=1, b^{2}=1, a b=a^{-1} b\right\rangle .
$$

Definition 3 [9] $p$ - group

A $p$-group is defined as the group that has its order as prime power, labeled as $p^{n}$, where $p$ is prime and $n$ is non-negative integers.

Definition 4 [2] Let $G$ be a finite group. For any $x, y \in G$, the co-prime probability of $G$, denoted as $P_{\text {copr }}(G)$ is defined as:

$$
P_{\text {copr }}(G)=\frac{|\{(x, y) \in G \times G,(|x|,|y|)=1\}|}{|G|^{2}} .
$$


Proposition 1 [2] Let $G$ be a finite $p$-group of order $p^{n}$ where $n \geq 1$. Then

$$
P_{\text {copr }}(G)=\frac{2 p^{n}-1}{p^{2 n}} \text {. }
$$

Proposition 2 [2] Let $D_{n}$ be a dihedral group of order $2 n$, where $n \geq 3$ and $n$ is odd. Then

$$
P_{\text {copr }}(G)=\frac{2 n^{2}+2 n-1}{4 n^{2}}
$$

Definition 5 [10] A graph $\Gamma$ is connected if each pair of the vertices are joined by a path.

Definition 6 [10] The degree of vertex $x$, deg $(x)$ is the number of edges incident with $x$, each loop counting as two edges.

Definition 7 [10] The dominating set $X \subseteq V(\Gamma)$ is a set where for each $v$ outside $W$ there exist $x \in X$ such that $v$ is adjacent to $x$. The minimum size of $x$ is called the dominating number and it is denoted by $\gamma(\Gamma)$.

Definition 8 [10] A non-empty $S$ of $V(\Gamma)$ is called an independent set of $\Gamma$ if there is no adjacent between two elements of $S$ in $\Gamma$. Thus the independent number is the number of vertices in maximum independent set and it is denoted as $\alpha(\Gamma)$.

Definition 9 [11] A star graph $K_{1, n}$ is a graph which consists of a single vertex with $n$ neighbours.

Definition 10 [11] A planar graph is a graph that can be drawn in a plane, so that no two lines intersect except at the vertices; otherwise it is said to be non-planar.

Definition 11 [11] A bipartite graph or a bigraph, $K_{m, n}$ is a set of vertices partitioned in to subsets such that there is no adjacent between two graph vertices within the same set.

Definition 12 [12] The complete tripartite graph, $K_{r, s, t}$ consist of three sets (of sizes $r, s, t$ ) with edges joining two vertices if and only if they lie in different set.

Definition 13 [6] The co-prime graph of $G$ denoted as $\Gamma$ is a graph whose vertices are elements of $G$ and two distinct vertices $u$ and $v$ are adjacent if and only of $(|u|,|v|)=1$.

Proposition 3 [6] Let $G$ be a group. Then, $G$ is not a $p$-groups if and only if $\Gamma_{G}$ is not bipartite.

Proposition 4 [6] Let $G$ be a group. If $G$ is cyclic with order $2 p$ for some odd prime $p$, then $\Gamma_{G}$ is planar.

Proposition 5 [6] Let $p$ and $q$ be two distinct prime numbers and $G$ be a non-cyclic group with order $p g$. Then $\Gamma_{G}$ is a complete tripartite graph. 
Theorem 1 [6] Let $\Gamma_{D_{2 n}}$ be the co-prime graph of $D_{2 n}$ and let $n$ be odd. Then

(1) $\operatorname{deg}_{\Gamma_{D_{2 n}}}\left(s r^{i}\right)=n$ for any $1 \leq i \leq n$;

(2) $\operatorname{deg}_{\Gamma_{D_{2 n}}}\left(r^{i}\right) \leq n$ for any $1 \leq i \leq n$;

(3) $\Gamma_{D_{2 n}}$ is not Eulerian;

(4) $\Gamma_{D_{2 n}}$ is Hamiltonian;

(5) $\Gamma_{D_{2 n}}$ is not planar.

Proposition 6 [6] Let $G$ be a group with order greater than 2. Then, $e$ is unique dominating set of size 1 of $\Gamma_{G}$. In particular, $\gamma(\Gamma)=1$ and $\operatorname{deg}_{\Gamma_{G}}(e)=|G|-1$.

\section{Co-prime Probability with Their Related Graphs for Nonabelian Metabelian Groups of Order Less than 24}

The first part of this section focuses on computing the co-prime probability for nonabelian metabelian groups of order less than 24 meanwhile the second part explains the co-prime graph, their types and the properties of the graph. As for the properties of the graph, the focus is on the dominating and independent number only.

\subsection{Co-prime Probability for Nonabelian Metabelian Groups of Order Less than 24}

In this subsection, the co-prime probability for nonabelian metabelian groups of order less than 24 are determined. Firstly, Cayley table generated by Maple 2016 software is used to find the order for each element in the group. Then, the co-prime probability is identified by applying Definition 4. The following four lemmas explain the finding of co-prime probability for nonabelian metabelian groups of order 12, 18, 20 and 21, respectively.

Lemma 1 Let $G$ be a nonabelian metabelian group of order 12. Then,

$$
P_{\text {copr }}(G)= \begin{cases}51 / 144, & \text { if } G=\mathbb{Z}_{3} \rtimes \mathbb{Z}_{4} \text { and } D_{6}, \\ 71 / 144, & \text { if } G=A_{4} .\end{cases}
$$

Proof Let $G=\mathbb{Z}_{3} \rtimes \mathbb{Z}_{4}$. Then, $G=\left\langle a, b \mid a^{4}=b^{3}=1, b a b=a\right\rangle=\left\{e, a, a^{2}, a^{3}, b, a b, a^{2} b, a^{3} b, b a\right.$, $\left.a b a, a^{2} b a, a^{3} b a\right\}$. Firstly, the Cayley table of $\mathbb{Z}_{3} \rtimes \mathbb{Z}_{4}$ is formed to determine the order for each element in $\mathbb{Z}_{3} \rtimes \mathbb{Z}_{4}$. The Cayley table of $\mathbb{Z}_{3} \rtimes \mathbb{Z}_{4}$ is shown in Figure 1 .

It can be found that $|e|=1,\left|a^{2}\right|=2,|b|=\left|a^{3} b a\right|=3,|a|=\left|a^{3}\right|=|a b|=\left|a^{3} b\right|=|b a|=$ $\left|a^{2} b a\right|=4$ and $\left|a^{2} b\right|=|a b a|=6$. In order to find the $P_{\text {copr }}(G)$, three cases that should be considered are given as follows:

Case 1 : If $x=e$, then $x$ is co-prime to each element $y$ in $G$ since $(|x|,|y|)=(|e|,|y|)=1$. Let $N_{1}=\{(e, y) \in G \times G\}$. So, $\left|N_{1}\right|=12$. Now, let $y=e$, then $y$ is also co-prime to each element $x$ in $G \backslash e$ since $(|x|,|y|)=(|x|,|e|)=1$. Let $N_{2}=\{(x, e) \in G \backslash e \times G\}$. So, $\left|N_{2}\right|=11$.

Case 2 : Let $N_{3}=\{(x, y) \in G \times G|| x|=2| y \mid,=3\}$, then $x=a^{2}$ and $y \in\left\{b, a^{3} b a\right\}$. This implies $\left|N_{3}\right|=2$. Next, let $N_{4}=\{(x, y) \in G \times G|| x|=3| y \mid,=2\}$, then $x \in\left\{b, a^{3} b a\right\}$ and $y=a^{2}$. Thus, $\left|N_{4}\right|=2$.

Case 3 : Let $N_{5}=\{(x, y) \in G \times G|| x|=3| y \mid,=4\}$, then $x \in\left\{b, a^{3} b a\right\}$ and $y \in$ 


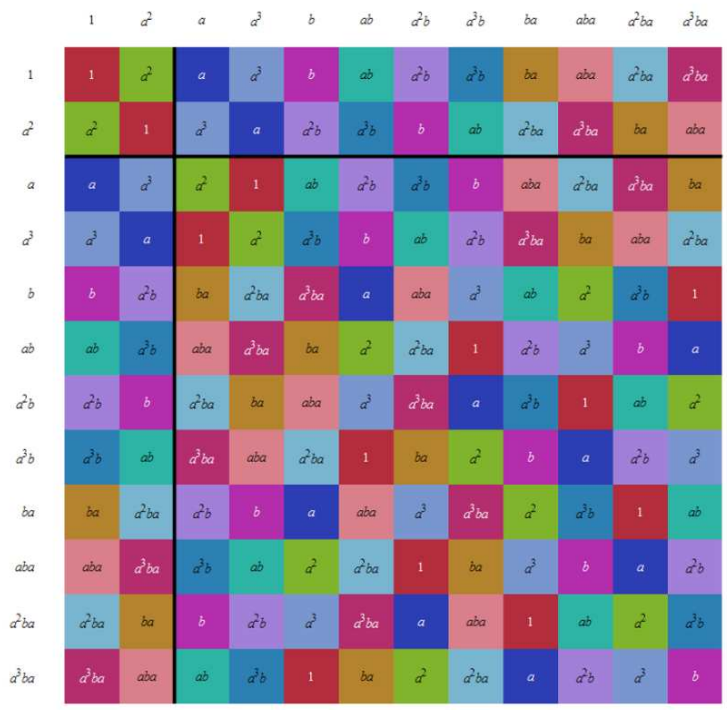

Figure 1: The Cayley Table of $\mathbb{Z}_{3} \rtimes \mathbb{Z}_{4}$

$\left\{a, a^{3}, a b, a^{3} b, b a, a^{2} b a\right\}$. This implies $\left|N_{5}\right|=12$. Now, let $N_{6}=\{(x, y) \in G \times G|| x|=4| y \mid,=3\}$, then $x \in\left\{a, a^{3}, a b, a^{3} b, b a, a^{2} b a\right\}$ and $y \in\left\{b, a^{3} b a\right\}$. Thus, $\left|N_{6}\right|=12$.

Hence,

$$
P_{\text {copr }}\left(\mathbb{Z}_{3} \rtimes \mathbb{Z}_{4}\right)=\frac{\left|N_{1}\right|+\left|N_{2}\right|+\left|N_{3}\right|+\left|N_{4}\right|+\left|N_{5}\right|+\left|N_{6}\right|}{\left|\mathbb{Z}_{3} \rtimes \mathbb{Z}_{4}\right|^{2}}=\frac{51}{144} .
$$

Now, let $G=D_{6}$. Then, $G=\left\langle a, b \mid a^{6}=b^{2}=1, b a b=a^{-1}\right\rangle=$ $\left\{e, a, a^{2}, a^{3}, a^{4}, a^{5}, b, a b, a^{2} b, a^{3} b, a^{4} b, a^{5} b\right\}$. By referring to Cayley table of $D_{6}$, it can be found that $|e|=1,\left|a^{3}\right|=|b|=|a b|=\left|a^{2} b\right|=\left|a^{3} b\right|=\left|a^{4} b\right|=\left|a^{5} b\right|=2,\left|a^{2}\right|=\left|a^{4}\right|=3$ and $|a|=\left|a^{5}\right|=6$. In order to find $P_{\text {copr }}(G)$, below are the cases that should be considered:

Case 1 : If $x=e$, then $x$ is co-prime to each element $y$ in $G$ since $(|x|,|y|)=(|e|,|y|)=1$. Let $N_{1}=\{(e, y) \in G \times G\}$. Then, $\left|N_{1}\right|=12$. Now, let $y=e$, then $y$ is also co-prime to all $x$ in $G \backslash e$ since $(|x|,|y|)=(|x|,|e|)=1$. Let $N_{2}=\{(x, e) \in G \backslash e \times G\}$.Then, $\left|N_{2}\right|=11$.

Case 2 : Let $N_{3}=\{(x, y) \in G \times G|| x|=2| y \mid,=3\}$, then $x \in\left\{a^{3}, b, a b, a^{2} b, a^{3} b, a^{4} b, a^{5} b\right\}$ and $y \in\left\{a^{2}, a^{4}\right\}$. This implies $\left|N_{3}\right|=14$. Next, let $N_{4}=\{(x, y) \in G \times G|| x|=3| y \mid,=2\}$, then $x \in\left\{a^{2}, a^{4}\right\}$ and $y \in\left\{a^{3}, b, a b, a^{2} b, a^{3} b, a^{4} b, a^{5} b\right\}$. Thus, $\left|N_{4}\right|=14$.

Hence,

$$
P_{\text {copr }}\left(D_{6}\right)=\frac{\left|N_{1}+N_{2}+N_{3}+N_{4}\right|}{\left|A_{4}\right|^{2}}=\frac{51}{144} .
$$

Next, let $G=A_{4}$. Then, $G=\left\langle a, b, c \mid a^{2}=b^{2}=c^{3}=1, b a=a b, c a=a b c, c b=a c\right\rangle=\{e, a, b, c$, $\left.c^{2}, a b, a c, a c^{2}, b c, b c^{2}, a b c, a b c^{2}\right\}$. By referring to Cayley table of $A_{4}$, the order of each element is $|e|=1,|a|=|b|=|a b|=2$ and $|c|=\left|c^{2}\right|=|a c|=\left|a c^{2}\right|=|b c|=\left|b c^{2}\right|=|a b c|=\left|a b c^{2}\right|=3$. The co-prime probability of $G$ is calculated as follows:

Case 1 : If $x=e$, then $x$ is co-prime to each element $y$ in $G$ since $(|x|,|y|)=(|e|,|y|)=1$. Let $N_{1}=\{(e, y) \in G \times G\}$. Then, $\left|N_{1}\right|=12$. Now, if $y=e$, then $y$ is also co-prime to all $x$ in $G \backslash e$ since $(|x|,|y|)=(|x|,|e|)=1$. Let $N_{2}=\{(x, e) \in G \backslash e \times G\}$. Then, $\left|N_{2}\right|=11$. 
Case 2 : Let $N_{3}=\{(x, y) \in G \times G|| x|=2| y \mid,=3\}$, then $x \in\{a, b, a b\}$ and $y \in\left\{c, c^{2}, a c, a c^{2}, b c, b c^{2}, a b c, a b c^{2}\right\}$. This implies $\left|N_{3}\right|=24$. Next, let $N_{4}=\{(x, y) \in G \times G \mid$ $|x|=3,|y|=2\}$, then $x \in\left\{c, c^{2}, a c, a c^{2}, b c, b c^{2}, a b c, a b c^{2}\right\}$ and $y \in\{a, b, a b\}$. Thus, $\left|N_{4}\right|=24$.

Hence,

$$
P_{\text {copr }}\left(A_{4}\right)=\frac{\left|N_{1}\right|+\left|N_{2}\right|+\left|N_{3}\right|+\left|N_{4}\right|}{\left|A_{4}\right|^{2}}=\frac{71}{144} .
$$

Lemma 2 Let $G$ be nonabelian metabelian group of order 18. Then,

$$
P_{\text {copr }}(G)= \begin{cases}83 / 324, & \text { if } G=S_{3} \times \mathbb{Z}_{3}, \\ 179 / 324, & \text { if } G=\left(\mathbb{Z}_{3} \times \mathbb{Z}_{3}\right) \rtimes \mathbb{Z}_{2} .\end{cases}
$$

Proof Let $G=S_{3} \times \mathbb{Z}_{3}$. Then, $G=\left\langle a, b, c \mid a^{3}=b^{2}=c^{3}=1, b a b=a^{-1}, a c=c a, b c=c b\right\rangle=$ $\left\{e, a, a^{2}, \quad b, c, c^{2}, a b, a^{2} b, a c, a c^{2}, a^{2} c, a^{2} c^{2}, b c, b c^{2}, a b c, a b c^{2}, a^{2} b c, a^{2} b c^{2}\right\} . \quad$ By $\quad$ referring to Cayley table of $S_{3} \times \mathbb{Z}_{3}$, the order for each element in $S_{3} \times \mathbb{Z}_{3}$ are $|e|=1$, $|b|=|a b|=\left|a^{2} b\right|=2,|a|=\left|a^{2}\right|=|c|=\left|c^{2}\right|=|a c|=\left|a c^{2}\right|=\left|a^{2} c\right|=\left|a^{2} c^{2}\right|=3$ and $|b c|=\left|b c^{2}\right|=|a b c|=\left|a b c^{2}\right|=\left|a^{2} b c\right|=\left|a^{2} b c^{2}\right|=6$. Below are the cases that should be considered in order to find $P_{\text {copr }}(G)$ :

Case 1 : If $x=e$, then $x$ is co-prime to each element $y$ in $G$ since $(|x|,|y|)=(|e|,|y|)=1$. Let $N_{1}=\{(e, y) \in G \times G\}$. Then, $\left|N_{1}\right|=18$. Now, if $y=e$, then $y$ is also co-prime to all $x$ in $G \backslash e$ since $(|x|,|y|)=(|x|,|e|)=1$. Let $N_{2}=\{(x, e) \in G \backslash e \times G\}$. Then, $\left|N_{2}\right|=17$.

Case 2 : Let $N_{3}=\{(x, y) \in G \times G|| x|=2| y \mid,=3\}$, then $x \in\left\{b, a b, a^{2} b\right\}$ and $y \in\left\{a, a^{2}, c, c^{2}, a c, a c^{2}, a^{2} c, a^{2} c^{2}\right\}$. This implies $\left|N_{3}\right|=24$. Next, let $N_{4}=\{(x, y) \in G \times G \mid$ $|x|=3,|y|=2\}$, then $x \in\left\{a, a^{2}, c, c^{2}, a c, a c^{2}, a^{2} c, a^{2} c^{2}\right\}$ and $y \in\left\{b, a b, a^{2} b\right\}$. Thus, $\left|N_{4}\right|=24$. Hence,

$$
P_{\text {copr }}\left(S_{3} \times \mathbb{Z}_{3}\right)=\frac{\left|N_{1}\right|+\left|N_{2}\right|+\left|N_{3}\right|+\left|N_{4}\right|}{\left|S_{3} \times \mathbb{Z}_{3}\right|^{2}}=\frac{83}{324} .
$$

Next, let $G=\left(\mathbb{Z}_{3} \times \mathbb{Z}_{3}\right) \rtimes \mathbb{Z}_{2}$. Then, $G=\left\langle a, b, c \mid a^{2}=b^{3}=c^{3}=1, b c=c b, b a b=a, c a c=a\right\rangle=$ $\{e, a, b, a b a, c, a c a, a b, b a, a c, c a, b c, b a c a, a b a c, a b c a, a b c, a b a c a, b a c, b c a\}$. So, from the Cayley table of $\left(\mathbb{Z}_{3} \times \mathbb{Z}_{3}\right) \rtimes \mathbb{Z}_{2}$, it can be found that $|e|=1,|a|=|a b|=|b a|=|a c|=|c a|=|a b c|=$ $|a b a c a|=|b a c|=|b c a|=2$ and $|b|=|a b a|=|c|=|a c a|=|b c|=|b a c a|=|a b a c|=|a b c a|=3$. Two cases that need to be considered to find the co-prime probability of $G$ are as follows:

Case 1 : If $x=e$, then $x$ is co-prime to each element $y$ in $G$ since $(|x|,|y|)=(|x|,|y|)=1$. Let $N_{1}=\{(e, y) \in G \times G\}$. Then, $\left|N_{1}\right|=18$. Now, if $y=e$, then $y$ is also co-prime to all $x$ in $G \backslash e$ since $(|x|,|y|)=(|x|,|e|)=1$. Let $N_{2}=\{(x, e) \in G \backslash e \times G\}$. Then, $\left|N_{2}\right|=17$.

Case 2 : Let $N_{3}=\{(x, y) \in G \times G|| x|=2| y \mid,=3\}$, then $x \in\{a, a b, b a, a c, c a, a b c, a b a c a, b a c$, $b c a\}$ and $y \in\{b, a b a, c, a c a, b c, b a c a, a b a c, a b c a\}$. This implies $\left|N_{3}\right|=72$. Next, let $N_{4}=$ $\{(x, y) \in G \times G|| x|=3| y \mid,=2\}$, then $x \in\{b, a b a, c, a c a, b c, b a c a, a b a c, a b c a\}$ and $y \in$ $\{a, a b, b a, a c, c a, a b c, a b a c a, b a c, b c a\}$. Thus, $\left|N_{4}\right|=72$.

Hence,

$$
P_{\text {copr }}\left(\left(\mathbb{Z}_{3} \times \mathbb{Z}_{3}\right) \rtimes \mathbb{Z}_{2}\right)=\frac{\left|N_{1}\right|+\left|N_{2}\right|+\left|N_{3}\right|+\left|N_{4}\right|}{\left|\left(\mathbb{Z}_{3} \times \mathbb{Z}_{3}\right) \rtimes \mathbb{Z}_{2}\right|^{2}}=\frac{179}{324} .
$$


Lemma 3 Let $G$ be nonabelian metabelian group of order 20. Then,

$$
P_{\text {copr }}(G)=\frac{127}{400}, \text { if } G=D_{10}, F r_{20} \cong \mathbb{Z}_{5} \rtimes \mathbb{Z}_{4} \text { and } \mathbb{Z}_{5} \rtimes \mathbb{Z}_{5}
$$

Proof Suppose $G$ is nonabelian metabelian group of order 20. Now, let $G=D_{10}$ and $G=\left\langle a, b \mid a^{10}=b^{2}=1, b a b=a^{-1}\right\rangle=$ $\left\{e, a, a^{2}, a^{3}, a^{4}, a^{5}, a^{6}, a^{7}, a^{8}, a^{9}, b, a b, a^{2} b, a^{3} b, a^{4} b, a^{5} b, a^{6} b, a^{7}, a^{8} b, a^{9} b\right\}$. By referring to Cayley table of $D_{10}$, the order for each element is $|e|=1,\left|a^{5}\right|=|b|=|a b|=\left|a^{2} b\right|=\left|a^{3} b\right|=$ $\left|a^{4} b\right|=\left|a^{5} b\right|=\left|a^{6} b\right|=\left|a^{7} b\right|=\left|a^{8} b\right|=\left|a^{9} b\right|=2,\left|a^{2}\right|=\left|a^{4}\right|=\left|a^{6}\right|=\left|a^{8}\right|=5$ and $|a|=\left|a^{3}\right|=\left|a^{7}\right|=\left|a^{9}\right|=10$. In order to find $P_{\text {copr }}(G)$, below are the cases that should be considered:

Case 1 : If $x=e$, then $x$ is co-prime to each element $y$ in $G$ since $(|x|,|y|)=(|e|,|y|)=1$. Let $N_{1}=\{(e, y) \in G \times G\}$. Then, $\left|N_{1}\right|=20$. Now, if $y=e$, then $y$ is also co-prime to all $x$ in $G \backslash e$ since $(|x|,|y|)=(|x|,|e|)=1$. Let $N_{2}=\{(x, e) \in G \backslash e \times G\}$. Then, $\left|N_{2}\right|=19$.

Case 2 : Let $N_{3}=\{(x, y) \in G \times G|| x|=2| y \mid,=5\}$, then $x \in$ $\left\{a^{5}, b, a b, a^{2} b, a^{3} b, a^{4} b, a^{5} b, a^{6} b, a^{7} b, a^{8} b, a^{9} b\right\}$ and $y \in\left\{a^{2}, a^{4}, a^{6}, a^{8}\right\}$. This implies $\left|N_{3}\right|=44$. Next, let $N_{4}=\{(x, y) \in G \times G|| x|=5| y \mid,=2\}$, then $x \in\left\{a^{2}, a^{4}, a^{6}, a^{8}\right\}$ and $y \in\left\{a^{5}, b, a b, a^{2} b, a^{3} b, a^{4} b, a^{5} b, a^{6} b, a^{7}, a^{8} b, a^{9} b\right\}$. Thus, $\left|N_{4}\right|=44$. Hence,

$$
P_{\text {copr }}\left(D_{10}\right)=\frac{\left|N_{1}\right|+\left|N_{2}\right|+\left|N_{3}\right|+\left|N_{4}\right|}{\left|D_{10}\right|^{2}}=\frac{127}{400} .
$$

Next, let $G=F r_{20} \cong \mathbb{Z}_{5} \rtimes \mathbb{Z}_{4}$. So, $G=\left\langle a, b \mid a^{5}=b^{4}=1, a b a=b\right\rangle=\left\{e, a, a^{2}, a^{3}, a^{4}, b, b^{2}, b^{3}, a b\right.$, $\left.a b^{2}, a b^{3}, a^{2} b, a^{2} b^{2}, a^{2} b^{3}, a^{3} b, a^{3} b^{2}, a^{3} b^{3}, a^{4} b, a^{4} b^{2}, a^{4} b^{3}\right\}$. Then, the order for each element is $|e|=1,\left|b^{2}\right|=2,|b|=\left|b^{3}\right|=|a b|=\left|a b^{3}\right|=\left|a^{2} b\right|=\left|a^{2} b^{3}\right|=\left|a^{3} b\right|=\left|a^{3} b^{3}\right|=\left|a^{4} b\right|=\left|a^{4} b^{3}\right|=4$, $\left|a^{2}\right|=\left|a^{4}\right|=|a|=\left|a^{3}\right|=5$ and $\left|a b^{2}\right|=\left|a^{2} b^{2}\right|=\left|a^{3} b^{2}\right|=10$. Below are the cases that should be considered:

Case 1 : If $x=e$, then $x$ is co-prime to each element $y$ in $G$, since $(|x|,|y|)=(|e|,|y|)=1$. Let $N_{1}=\{(e, y) \in G \times G\}$. Then, $\left|N_{1}\right|=20$. Now, if $y=e$, then $y$ is also co-prime to all $x$ in $G \backslash e$ since $(|x|,|y|)=(|x|,|e|)=1$. Let $N_{2}=\{(x, e) \in G \backslash e \times G\}$.Then, $\left|N_{2}\right|=19$.

Case 2 : Let $N_{3}=\{(x, y) \in G \times G|| x|=2| y \mid,=5\}$, then $x \in\left\{b^{2}\right\}$ and $y \in\left\{a^{2}, a^{4}, a, a^{3}\right\}$. This implies $\left|N_{3}\right|=4$. Next, let $N_{4}=\{(x, y) \in G \times G|| x|=5| y \mid,=2\}$, then $x \in\left\{a^{2}, a^{4}, a, a^{3}\right\}$ and $y \in\left\{b^{2}\right\}$. Thus, $\left|N_{4}\right|=4$.

Case 3 : Let $N_{5}=\{(x, y) \in G \times G|| x|=4| y \mid,=5\}$, then $x \in\left\{b, b^{3}, a b, a b^{3}, a^{2} b, a^{2} b^{3}, a^{3} b, a^{3} b^{3}\right.$, $\left.a^{4} b, a^{4} b^{3}\right\}$ and $y \in\left\{a^{2}, a^{4}, a, a^{3}\right\}$. This implies $\left|N_{5}\right|=40$. Now, let $N_{6}=\{(x, y) \in G \times G \mid$ $|x|=5,|y|=4\}$, then $x \in\left\{a^{2}, a^{4}, a, a^{3}\right\}$ and $y \in\left\{b, b^{3}, a b, a b^{3}, a^{2} b, a^{2} b^{3}, a^{3} b, a^{3} b^{3}, a^{4} b, a^{4} b^{3}\right\}$. Thus, $\left|N_{6}\right|=40$. Hence,

$$
P_{\text {copr }}\left(F r_{20} \cong \mathbb{Z}_{5} \rtimes \mathbb{Z}_{4}\right)=\frac{\left|N_{1}\right|+\left|N_{2}\right|+\left|N_{3}\right|+\left|N_{4}\right|+\left|N_{5}\right|+\left|N_{6}\right|}{\left|F r_{20} \cong \mathbb{Z}_{5} \rtimes \mathbb{Z}_{4}\right|^{2}}=\frac{127}{400}
$$

Now, let $G=\mathbb{Z}_{5} \rtimes \mathbb{Z}_{5}$ where $G=\left\langle a, b \mid a^{5}=b^{4}=1, a b a=b\right\rangle=\left\{e, a, a^{2}, a^{3}, b, b^{2}, a^{3} b^{2} a, a^{3} b a, a b\right.$, $\left.a b^{2}, b^{2} a, b a, a^{2} b, a^{2} b^{2}, a b^{2} a, a b a, a^{3} b, a^{3} b^{2}, a^{2} b^{2} a, a^{2} b a\right\}$. Therefore, from the Cayley table of $\mathbb{Z}_{5} \rtimes$ $\mathbb{Z}_{5}$, it can be found that $|e|=1,\left|a^{2}\right|=2,|a|=\left|a^{3}\right|=|a b|=\left|a b^{2}\right|=\left|a b^{3}\right|=\left|a b^{4}\right|=\left|a^{3} b\right|=$ 
$\left|a^{3} b^{2}\right|=\left|a^{3} b^{3}\right|=\left|a^{3} b^{4}\right|=4,\left|b^{2}\right|=|b|=\left|a^{3} b a\right|=\left|a^{3} b^{2} a\right|=5$ and $\left|a^{2} b\right|=\left|a^{2} b^{2}\right|=\left|a b^{2} a\right|=$ $|a b a|=10$. In order to find $P_{\text {copr }}(G)$, below are the cases that should be considered:

Case 1 : If $x=e$, then $x$ is co-prime to each element $y$ in $G$ since $(|x|,|y|)=(|e|,|y|)=1$. Let $N_{1}=\{(e, y) \in G \times G\}$. Then, $\left|N_{1}\right|=20$. Now, if $y=e$, then $y$ is also co-prime to each element $x$ in $G \backslash e$ since $(|x|,|y|)=(|x|,|e|)=1$. Let $N_{2}=\{(x, e) \in G \backslash e \times G\}$. Then, $\left|N_{2}\right|=19$.

Case 2 : Let $N_{3}=\{(x, y) \in G \times G|| x|=2| y \mid,=5\}$, then $x \in\left\{a^{2}\right\}$ and $y \in\left\{b^{2}, b, a^{3} b a, a^{3} b^{2} a\right\}$. This implies $\left|N_{3}\right|=4$. Next, let $N_{4}=\{(x, y) \in G \times G|| x|=5| y \mid,=2\}$, then $x \in$ $\left\{b^{2}, b, a^{3} b a, a^{3} b^{2} a\right\}$ and $y \in\left\{a^{2}\right\}$. Thus, $\left|N_{4}\right|=4$.

Case 3 : Let $N_{5}=\{(x, y) \in G \times G|| x|=4| y \mid,=5\}$, then $x \in\left\{a, a^{3}, a b, a b^{2}, a b^{3}, a b^{4}, a^{3} b, a^{3} b^{2}\right.$, $\left.a^{3} b^{3}, a^{3} b^{4}\right\}$ and $y \in\left\{b^{2}, b, a^{3} b a, a^{3} b^{2} a\right\}$. This implies $\left|N_{5}\right|=40$. Now, Let $N_{6}=$ $\{(x, y) \in G \times G|| x|=5| y \mid,=4\}$, then $x \in\left\{b^{2}, b, a^{3} b a, a^{3} b^{2} a\right\}$ and $y \in$ $\left\{a, a^{3}, a b, a b^{2}, a b^{3}, a b^{4}, a^{3} b, a^{3} b^{2}, a^{3} b^{3}, a^{3} b^{4}\right\}$. Thus, $\left|N_{6}\right|=40$. Hence,

$$
P_{\text {copr }}\left(\mathbb{Z}_{5} \rtimes \mathbb{Z}_{5}\right)=\frac{\left|N_{1}\right|+\left|N_{2}\right|+\left|N_{3}\right|+\left|N_{4}\right|+\left|N_{5}\right|+\left|N_{6}\right|}{\left|\mathbb{Z}_{5} \rtimes \mathbb{Z}_{5}\right|^{2}}=\frac{127}{400} .
$$

Lemma 4 Let $G$ be nonabelian metabelian group of order 21. Then,

$$
P_{\text {copr }}(G)=\frac{209}{441}
$$

Proof Suppose $G$ is nonabelian metabelian group of order 21 where $G=F r_{21} \cong \mathbb{Z}_{7} \rtimes \mathbb{Z}_{3}$. Then, $G=\left\langle a, b \mid a^{3}=b^{7}=1, b a=a b^{2}\right\rangle=\left\{e, a, a^{2}, b, a^{2} b a, a^{2} b a b, a b a^{2}, a b a^{2} b, a b a b a, a b, b a, b a b, a^{2} b a^{2}\right.$, $\left.a^{2} b a^{2} b, a^{2} b a b a, a^{2} b, a b a, a b a b, b a^{2}, b a^{2} b, b a b a\right\}$. By referring to Cayley table of $F r_{21} \cong \mathbb{Z}_{7} \rtimes \mathbb{Z}_{3}$, it can be seen that $|e|=1,|a|=\left|a^{2}\right|=|a b|=|b a|=|b a b|=\left|a^{2} b a^{2}\right|=$ $\left|a^{2} b a^{2} b\right|=\left|a^{2} b a b a\right|=\left|a^{2} b\right|=|a b a|=|a b a b|=\left|b a^{2}\right|=\left|b a^{2} b\right|=|b a b a|=3$ and $|b|=\left|a^{2} b a\right|=\left|a^{2} b a b\right|=\left|a b a^{2}\right|=\left|a b a^{2} b\right|=|a b a b a|=7$. The co-prime probability of $G$ are calculated as follows:

Case 1 : If $x=e$, then $x$ is co-prime to each element $y$ in $G$ since $(|x|,|y|)=(|e|,|y|)=1$. Let $N_{1}=\{(e, y) \in G \times G\}$. Then, $\left|N_{1}\right|=21$. Now, if $y=e$, then $y$ is also co-prime to all $x$ in $G \backslash e$ since $(|x|,|y|)=(|x|,|e|)=1$. Let $N_{2}=\{(x, e) \in G \backslash e \times G\}$.Then, $\left|N_{2}\right|=20$.

Case 2 : Let $N_{3}=\{(x, y) \in G \times G|| x|=7| y \mid,=3\}$, then $x \in\left\{b, a^{2} b a, a^{2} b a b, a b a^{2}, a b a^{2} b, a b a b a\right\}$ and $y \in\left\{a, a^{2}, a b, b a, b a b, a^{2} b a^{2}, a^{2} b a^{2} b, a^{2} b a b a, \quad a^{2} b, a b a, a b a b, b a^{2}, b a^{2} b, b a b a\right\}$. This implies $\left|N_{3}\right|=84$. Next, let $N_{4}=\{(x, y) \in G \times G|| x|=3| y \mid,=7\}$, then $x \in\left\{a, a^{2}, a b, b a, b a b, a^{2} b a^{2}, a^{2} b a^{2} b, \quad a^{2} b a b a, a^{2} b, a b a, a b a b, b a^{2}, b a^{2} b, b a b a\right\}$ and $y \in\left\{b, a^{2} b a, a^{2} b a b, a b a^{2}, a b a^{2} b, a b a b a\right\}$. Thus, $\left|N_{4}\right|=84$.

Hence,

$$
P_{\text {copr }}\left(F r_{21} \cong \mathbb{Z}_{7} \rtimes \mathbb{Z}_{3}\right)=\frac{\left|N_{1}\right|+\left|N_{2}\right|+\left|N_{3}\right|+\left|N_{4}\right|}{\left|F r_{21} \cong \mathbb{Z}_{7} \rtimes \mathbb{Z}_{3}\right|^{2}}=\frac{209}{441} .
$$

Next, from the previous lemmas and propositions by Abd Rhani in [2], Theorem 2 is then giving the co-prime probability for nonabelian metabelian groups of order less than 24 . 


\section{Theorem 2}

Let $G$ be nonabelian metabelian group of order at most 24. Then,

$$
P_{\text {copr }}(G)= \begin{cases}23 / 36, & \text { if }|G|=6, \\ 15 / 64, & \text { if }|G|=8, \\ 59 / 100, & \text { if }|G|=10, \\ 51 / 144 \text { or } 71 / 144, & \text { if }|G|=12, \\ 111 / 196, & \text { if }|G|=14, \\ 31 / 256, & \text { if }|G|=16, \\ 179 / 324 \text { or } 83 / 324, & \text { if }|G|=18, \\ 127 / 400, & \text { if }|G|=20, \\ 209 / 441, & \text { if }|G|=21, \\ 263 / 484, & \text { if }|G|=22 .\end{cases}
$$

Proof The co-prime probability of these groups is proved by using Proposition 1 and 2 which have been determined by Abd Rhani in [2] whereas Lemma 1, 2, 3 and 4 are proved by using Definition 4 which is also has been determined by Abd Rhani [2]. Therefore, when $|G|=6$, 10,14 , and 22, the co-prime probability of $G$ are proven by Proposition 2. When $|G|=8$ and 16, Proposition 1 proved the co-prime probability of $G$. Next, when $|G|=12,20$ and 21, the co-prime probability of $G$ are proven by Lemma 1, Lemma 2 and Lemma 4 respectively. Lastly, when $|G|=18$, the co-prime probability for groups is proven by using Lemma 3 and Proposition 2.

\subsection{Co-prime Graph for Nonabelian Metabelian Groups of Order Less than 24}

In 2014, Ma et al. [6] have proven the co-prime graph, their types and properties, where the focus is on the finite group. So, a thorough understanding can be attained as the focus of this paper concentrates only on nonabelian metabelian groups of order less than 24 . Therefore, the co-prime graph, their types and the dominating number are explained. Apart from that, the independent number will also be determined as Ma et al. [6] did none on the independent number. The co-prime graph, the types of the graph and the properties of the graph is explained next when $G=D_{4}, D_{5}$ and $A_{4}$. Here, $D_{4}, D_{5}$ and $A_{4}$ are chosen as examples of types of groups where $D_{4}, D_{5}$ and $A_{4}$ is a $p$ - group, dihedral group or neither $p$-group nor dihedral group respectively. These groups are used to determine the graphs, the types of graph, the number of edges, the dominating and the independent number.

\subsubsection{The Co-prime Graph of $D_{4}$}

Let $D_{4}=\left\langle a, b \mid a^{4}=b^{2}=1, b a b=a^{-1}\right\rangle=\left\{e, a, a^{2}, a^{3}, b, a b, a^{2} b, a^{3} b\right\}$ and $\left|D_{4}\right|=8=2^{3}$. Therefore, the co-prime graph is illustrated below.

Hence, the total number of edges that connect to every vertex is 7 . It can also be concluded that the graph is a star which is also a bipartite graph from Proposition 3 as well as planar by Definition 10. Also, for the dominating number, $\gamma\left(\Gamma_{\text {copr }}\left(D_{4}\right)\right)=1$ as it follows from Proposition 6. As for the independent number, from Definition 8, the possible independent sets are as 


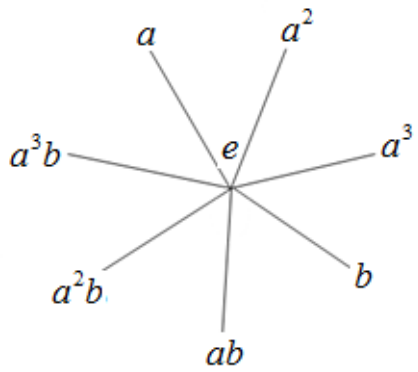

Figure 2: The Co-prime Graph of $D_{4}$

follows;

Size $2:\left\{a, a^{3}\right\},\left\{a, a^{2}\right\},\{a, b\}, \ldots$

Size $3:\left\{a, a^{2}, a^{3}\right\},\left\{a, a^{2}, b\right\},\left\{a, a^{2}, a b\right\}, \ldots$

Size $4:\left\{a, a^{2}, a^{3}, b\right\},\left\{a, a^{2}, a^{3}, a^{2} b\right\},\left\{a, a^{2}, a^{3}, a^{3} b\right\}, \ldots$

Size $5:\left\{a, a^{2}, a^{3}, b, a b\right\},\left\{a, a^{2}, a^{3}, b, a^{2} b\right\},\left\{a, a^{2}, a^{3}, b, a^{3} b\right\}, \ldots$

Size $6:\left\{a, a^{2}, a^{3}, b, a b, a^{2} b\right\},\left\{a, a^{2}, a^{3}, b, a b, a^{3} b\right\},\left\{a^{2}, a^{3}, b, a b, a^{2} b, a^{3} b\right\}, \ldots$

Size $7:\left\{a, a^{2}, a^{3}, b, a b, a^{2} b, a^{3} b\right\}$.

Therefore, $\alpha(\Gamma)=7$ since the largest independent set is $\left\{a, a^{2}, a^{3}, b, a b, a^{2} b, a^{3} b\right\}$. The same steps are repeated when $|G|=8$ and 16 as $G$ is a $p$-group of order $p^{n}$, where $n \geq 1$.

\subsubsection{The Co-prime Graph of $D_{5}$}

Let $D_{5}=\left\langle a, b \mid a^{5}=b^{2}=1, b a b=a^{-1}\right\rangle=\left\{e, a, a^{2}, a^{3}, a^{4}, b, a b, a^{2} b, a^{3} b, a^{4} b\right\}$ and $D_{5}$ is a dihedral group with $n=5$. Therefore, the co-prime graph is illustrated below.

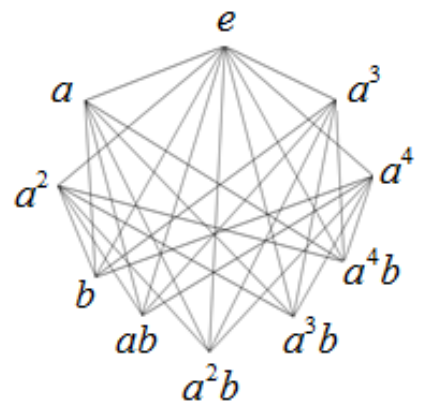

Figure 3: The Co-prime Graph of $D_{5}$

Hence, the total number of edges that connect to every vertex is 29 . It can also be concluded that the graph is a complete tripartite graph by using Proposition 5. Also, for the dominating number, $\gamma\left(\Gamma_{\text {copr }}\left(D_{5}\right)\right)=1$ as it follows from Proposition 6 . As for the independent number, from Definition 8, the possible independent sets are as follows;

Size $2:\left\{a, a^{3}\right\},\left\{a, a^{4}\right\},\left\{a, a^{2}\right\}, \ldots$

Size $3:\left\{b, a b, a^{2} b\right\},\left\{b, a b, a^{3} b\right\},\left\{b, a b, a^{4} b\right\}, \ldots$

Size $4:\left\{b, a b, a^{2} b, a^{3} b\right\},\left\{b, a b, a^{2} b, a^{4} b\right\},\left\{a, a^{2}, a^{3}, a^{4}\right\}, \ldots$

Size $5:\left\{b, a b, a^{2} b, a^{3} b, a^{4} b\right\}$. 
Therefore, $\alpha(\Gamma)=5$ since the largest independent set is $\left\{b, a b, a^{2} b, a^{3} b, a^{4} b\right\}$. The same steps are repeated when $|G|=6,10,14,18$ and 22 as $G$ is a dihedral group, $D_{n}$, whereby $n \geq 3$ and $n$ is odd.

\subsubsection{The Co-prime Graph of $\boldsymbol{A}_{4}$}

Let $A_{4}=\left\langle a, b, c \mid a^{2}=b^{2}=c^{3}=1, b a=a b, c a=a b c, c b=a c\right\rangle=\left\{e, a, b, c, c^{2}, a b, a c, a c^{2}, b c, b c^{2}\right.$, $\left.a b c, a b c^{2}\right\}$. Therefore, the co-prime graph is illustrated below.

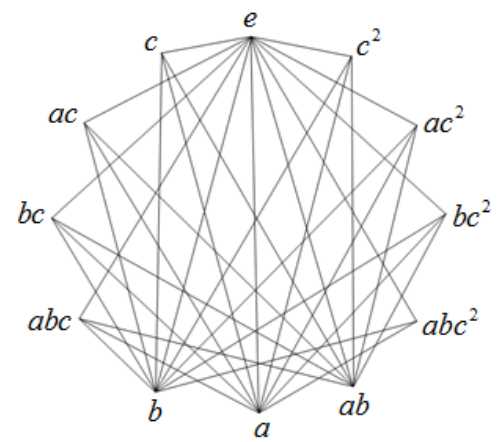

Figure 4: The Co-prime graph of $A_{4}$

Hence, the total number of edges that connect to every vertex is 35 . It can also be concluded that the graph is a complete tripartite graph based on Proposition 5. Also, for the dominating number, $\gamma\left(\Gamma_{\text {copr }}\left(A_{4}\right)\right)=1$ as it follows from Proposition 6 . As for the independent number, from Definition 8, the possible independent sets are as follows;

Size $2:\{c, a c\},\{c, b c\},\{a, b\}, \ldots$

Size $3:\{c, a c, b c\},\{c, a c, a b c\},\left\{c, a c, c^{2}\right\}, \ldots$

Size $4:\{c, a c, b c, a b c\},\left\{c, a c, b c, c^{2}\right\},\left\{c, a c, b c, a c^{2}\right\}, \ldots$

Size $5:\left\{c, a c, b c, a b c, c^{2}\right\},\left\{c, a c, b c, a b c, a c^{2}\right\},\left\{c, a c, b c, a b c, b c^{2}\right\}, \ldots$

Size $6:\left\{c, a c, b c, a b c, c^{2}, a c^{2}\right\},\left\{c, a c, b c, a b c, c^{2}, b c^{2}\right\},\left\{c, a c, b c, a b c, c^{2}, a b c^{2}\right\}, \ldots$

Size $7:\left\{c, a c, b c, a b c, c^{2}, a c^{2}, b c^{2}\right\},\left\{c, a c, b c, a b c, c^{2}, a c^{2}, a b c^{2}\right\}, \ldots$

Size $8:\left\{c, a c, b c, a b c, c^{2}, a c^{2}, b c^{2}, a b c^{2}\right\}$.

Therefore, $\alpha(\Gamma)=8$ since the largest independent set is $\left\{c, a c, b c, a b c, c^{2}, a c^{2}, b c^{2}, a b c^{2}\right\}$. Other groups that are neither $p$-group of order $p^{n}$ nor $G=D_{n}$ where $n \geq 3$ and $n$ is odd is proved as above.

The complete results of the co-prime graph for nonabelian metabelian groups of order less than 24 can be referred to Table 1. The table below indicates the number of edges, the types of graph, the dominating number and the independent number which used the definitions, some propositions and theorem by Ma et al. [6].

\section{Conclusion}

In this paper, the co-prime probability for nonabelian metabelian groups of order less than 24 and their related graphs whereby the number of edges, the types of graph together with its properties such as the dominating number and independent number are found. It can be 
concluded that the co-prime probability of dihedral groups or $p$ - group are the same when $G$ has the same order for nonabelian metabelian groups of order less than 24. Same goes to the number of edges and the independent number. When $G$ is neither dihedral group nor $p$-group for nonabelian metabelian groups of order less than 24 , the results for the co-prime probability, the number of edges and the independent number varies. Next, the dominating number for all groups for nonabelian metabelian groups of order less than 24 for the co-prime graph are equal to one. As for the types of graph, it can be concluded that when $G$ is a $p$ - group of order $p^{n}$, where $n \geq 1$, the types of graph are star, bipartite and planar. When $G=D_{n}$ with order $2 n$ where $n \geq 3$ and $n$ is odd, the types of graph are either complete tripartite or not bipartite, not planar, not Eulerian, but Hamiltonian. As for the other groups that are neither $p-$ group nor dihedral group, the types of graph are either not bipartite or complete tripartite

Table 1: Table of Co-prime Graph for Nonabelian Metabelian Groups of Order Less than 24

\begin{tabular}{|c|l|c|c|c|c|l|}
\hline No & $G$ & $|G|$ & $\begin{array}{c}\text { Number } \\
\text { of Edges }\end{array}$ & $\alpha(\Gamma)$ & $\gamma(\Gamma)$ & Type of graph \\
\hline 1 & $D_{3}$ & 6 & 11 & 1 & 3 & Complete tripartite \\
\hline 2 & $D_{4}$ & 8 & 7 & 1 & 7 & Star, bipartite, planar \\
\hline 3 & $Q=$ Quarternion & 8 & 7 & 1 & 7 & Star, bipartite, planar \\
\hline 4 & $D_{5}$ & 10 & 29 & 1 & 5 & Complete tripartite \\
\hline 5 & $\mathbb{Z}_{3} \rtimes \mathbb{Z}_{4}$ & 12 & 25 & 1 & 9 & Not bipartite \\
\hline 6 & $A_{4}$ & 12 & 35 & 1 & 8 & Complete tripartite \\
\hline 7 & $D_{6}$ & 12 & 25 & 1 & 9 & Not bipartite \\
\hline 8 & $D_{7}$ & 14 & 55 & 1 & 7 & Complete tripartite \\
\hline 9 & $D_{8}$ & 16 & 15 & 1 & 15 & Star, bipartite, planar \\
\hline 10 & Quasihedral-16 & 16 & 15 & 1 & 15 & Star, bipartite, planar \\
\hline 11 & $Q_{8}$ & 16 & 15 & 1 & 15 & Star, bipartite, planar \\
\hline 12 & $D_{4} \times \mathbb{Z}_{2}$ & 16 & 15 & 1 & 15 & Star, bipartite, planar \\
\hline 13 & $Q \times \mathbb{Z}_{2}$ & 16 & 15 & 1 & 15 & Star, bipartite, planar \\
\hline 14 & Modular-16 & 16 & 15 & 1 & 15 & Star, bipartite, planar \\
\hline 15 & $B$ & 16 & 15 & 1 & 15 & Star, bipartite, planar \\
\hline 16 & $K$ & 16 & 15 & 1 & 15 & Star, bipartite, planar \\
\hline 17 & $G_{4,4}$ & 16 & 15 & 1 & 15 & Star, bipartite, planar \\
\hline & & & & & & Not planar \\
18 & $D_{9}$ & 18 & 89 & 1 & 9 & Not Eulerian, not planar but Hamiltonian \\
& & & & & & Not bipartite \\
\hline 19 & $S_{3} \times \mathbb{Z}_{3}$ & 18 & 41 & 1 & 14 & Not bipartite \\
\hline 20 & $\left(\mathbb{Z}_{3} \times \mathbb{Z}_{3}\right) \rtimes \mathbb{Z}_{2}$ & 18 & 89 & 1 & 9 & Complete tripartite \\
\hline 21 & $D_{10}$ & 20 & 63 & 1 & 15 & Nor bipartite \\
\hline 22 & $F r_{20} \cong\left(\mathbb{Z}_{5} \rtimes \mathbb{Z}_{2}\right)$ & 20 & 63 & 1 & 15 & Nor bipartite \\
\hline 23 & $\mathbb{Z}_{5} \rtimes \mathbb{Z}_{5}$ & 20 & 63 & 1 & 15 & Nor bipartite \\
\hline 24 & $F r_{21} \cong\left(\mathbb{Z}_{7} \rtimes \mathbb{Z}_{3}\right)$ & 21 & 104 & 1 & 14 & Complete tripartite \\
\hline 25 & $D_{11}$ & 22 & 134 & 1 & 11 & Complete tripartite \\
\hline & & & & & \\
\hline
\end{tabular}

\section{References}

[1] Erdos, P. and Turan, P. On some problems of a statistical group theory IV. Acta Mathematica Hungarica. 1968. 19: 413-435. 
[2] Abd Rhani, N. Some Extensions of the Commutativity Degree and the Relative Co-prime Graph of Some Finite Groups. Ph.D. Thesis. Universiti Teknologi Malaysia. 2018.

[3] Williams, J. Prime graph components of finite groups. Journal of Algebra. 1981. 69(2): $487-513$.

[4] Erdos, P. and Sarkozy, G. N. On cycles in the co-prime graph of integers. Electron. J. Combin. 1997. 4(2): R8.

[5] Sarkozy, G. N. Complete tripartite subgraphs in the co-prime graph of integers. Discrete Math. 1999. 202: 227-238.

[6] Ma, X., Wei, H. and Yang, L. The co-prime graph of a group. International Journal of Group Theory. 2014. 3(3): 13-23.

[7] Wisnesky, R. J. Solvable group math 120. wisnesky.net/wim3.pdf. 2005.

[8] Dummit, D. S. and Foote, R. M. Abstract Algebra. Third Ed. John Wiley and Son, USA: 2004.

[9] Rotman, J. J. Advanced Modern Algebra. USA: Pearson Education. 2002.

[10] Bondy, J. A. and Murty, U. S. R. Graphs Theory (Graduate Texts in Mathematics). New York: Springer. 2008.

[11] Godsil, C. and Royle, G. Algebraic Graph Theory. 5th Edition Boston New York: Springer. 2001.

[12] Wilson, R. J. Introduction to Graph Theory. 2nd Edition London: Longman. 1979. 